ARTICLE

\title{
Crystallographic and electrophilic fragment screening of the SARS-CoV-2 main protease
}

\author{
Alice Douangamath (1) 1,2,11, Daren Fearon (1) 1,11, Paul Gehrtz 3,11, Tobias Krojer (1) 4,11, Petra Lukacik 1,2,11, \\ C. David Owen 1,2,11, Efrat Resnick 3,11, Claire Strain-Damerell,2,11, Anthony Aimon (1) 1,2, Péter Ábrányi-Balogh (1) 5, \\ José Brandão-Neto ${ }^{1,2}$, Anna Carbery 1,6, Gemma Davison ${ }^{7}$, Alexandre Dias', Thomas D. Downes (1) ${ }^{8}$, \\ Louise Dunnett ${ }^{1}$, Michael Fairhead ${ }^{4}$, James D. Firth (1) ${ }^{8}$, S. Paul Jones ${ }^{8}$, Aaron Keeley ${ }^{5}$, György M. Keserü ${ }^{5}$, \\ Hanna F. Klein ${ }^{8}$, Mathew P. Martin ${ }^{9}$, Martin E. M. Noble (10 9, Peter O'Brien (10 ${ }^{8}$, Ailsa Powell [0 1, \\ Rambabu N. Reddi (iD ${ }^{3}$, Rachael Skyner (10 1,2, Matthew Snee ${ }^{1}$, Michael J. Waring (1) ${ }^{7}$, Conor Wild ${ }^{1}$, \\ Nir London (10 ${ }^{3 凶}$, Frank von Delft (10) ${ }^{1,2,4,10 凶} \&$ Martin A. Walsh (10) $1,2 \otimes$
}

COVID-19, caused by SARS-CoV-2, lacks effective therapeutics. Additionally, no antiviral drugs or vaccines were developed against the closely related coronavirus, SARS-CoV-1 or MERS-CoV, despite previous zoonotic outbreaks. To identify starting points for such therapeutics, we performed a large-scale screen of electrophile and non-covalent fragments through a combined mass spectrometry and X-ray approach against the SARS-CoV-2 main protease, one of two cysteine viral proteases essential for viral replication. Our crystallographic screen identified 71 hits that span the entire active site, as well as 3 hits at the dimer interface. These structures reveal routes to rapidly develop more potent inhibitors through merging of covalent and non-covalent fragment hits; one series of low-reactivity, tractable covalent fragments were progressed to discover improved binders. These combined hits offer unprecedented structural and reactivity information for on-going structure-based drug design against SARS-CoV-2 main protease.

\footnotetext{
${ }^{1}$ Diamond Light Source Ltd., Harwell Science and Innovation Campus, Didcot OX11 OQX, UK. ${ }^{2}$ Research Complex at Harwell, Harwell Science and Innovation Campus, Didcot OX11 OFA, UK. ${ }^{3}$ Department of Organic Chemistry, Weizmann Institute of Science, Rehovot 7610001, Israel. ${ }^{4}$ Structural Genomics Consortium, University of Oxford, Old Road Campus, Roosevelt Drive, Headington OX3 7DQ, UK. ${ }^{5}$ Medicinal Chemistry Research Group, Research Centre for Natural Sciences, Magyar tudósok krt. 2, H-1117 Budapest, Hungary. ${ }^{6}$ Department of Statistics, University of Oxford, Oxford OX1 3LB, UK. ${ }^{7}$ Cancer Research UK Drug Discovery Unit, Newcastle University Centre for Cancer, Chemistry, School of Natural and Environmental Sciences, Bedson Building, Newcastle University, Newcastle upon Tyne NE1 7RU, UK. ${ }^{8}$ Department of Chemistry, University of York, Heslington, York YO10 5DD, UK. ${ }^{9}$ Cancer Research UK Drug Discovery Unit, Newcastle University Centre for Cancer, Paul O'Gorman Building, Medical School, Framlington Place, Newcastle University, Newcastle upon Tyne NE2 4AD, UK. ${ }^{10}$ Department of Biochemistry, University of Johannesburg, Auckland Park 2006, South Africa. ${ }^{11}$ These authors contributed equally: Alice Douangamath, Daren Fearon, Paul Gehrtz, Tobias Krojer, Petra Lukacik, C. David Owen,

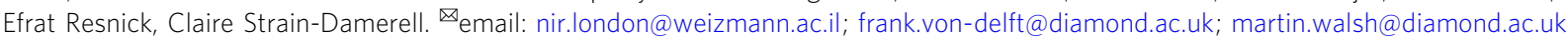


A novel coronavirus, SARS-CoV-2, the causative agent of COVID-19 $19^{1-3}$, has resulted in over one million confirmed cases and in excess of 300,000 deaths across 188 countries as of mid-May $2020^{4}$. SARS-CoV-2 is the third zoonotic coronavirus outbreak after the emergence of SARS-CoV-1 in 2002 and the Middle East Respiratory Syndrome (MERS-CoV) in $2012^{5-7}$. SARS-CoV-2 is a large enveloped, positive-sense, singlestranded RNA Betacoronavirus. The viral RNA encodes two open reading frames that, through ribosome frame-shifting, generates two polyproteins ppla and pplab ${ }^{8}$. These polyproteins produce most of the proteins of the replicase-transcriptase complex 9 . The polyproteins are processed by two viral cysteine proteases: a papain-like protease (PL ${ }^{\text {pro }}$ ) which cleaves three sites, releasing non-structural proteins nsp1-3 and a 3C-like protease, also referred to as the main protease $\left(\mathrm{M}^{\mathrm{pro}}\right)$, that cleaves at 11 sites to release non-structural proteins (nsp4-16). These non-structural proteins form the replicase complex responsible for replication and transcription of the viral genome and have led to Mpro and $\mathrm{PL}^{\text {Pro being the primary targets for antiviral drug development }}{ }^{10}$.

Structural biology, which can play a key role in drug development, was also rapidly deployed after the 2002 SARS-CoV-1 outbreak, with earlier work by the Hilgenfeld group on Mpro of coronarviruses $^{10}$ leading to crystal structures of SARS-CoV-1 $M^{\text {pro }}$ and inhibitor complexes ${ }^{1-14}$. Active sites of Coronavirus Mpro are well conserved ${ }^{13,15-19}$, and those of enteroviruses (3Cpro) are functionally similar: this underpins ambitions to develop broad-spectrum antivirals. The most successful have been peptidomimetic $\alpha$-ketoamide inhibitors ${ }^{20}$, with at least one potent variant seen as a potential antiviral drug ${ }^{19}$. Other studies have taken the popular approach of high-throughput screens (HTS) using very large compound libraries, followed by structural studies to elucidate the binding mode ${ }^{21}$.

Despite these efforts, drugs remain elusive that directly target SARS-CoV-2 (rather than disease symptoms) and are verified by clinical trials. In retrospect, this is perhaps unsurprising for the Mpro inhibitors, as both peptidomimetic and covalent inhibition carry risks as strategies for drug development; in general, the simpler the molecule, the lower the risk.

We therefore applied a different approach to Mpro, using fragment screening by high-throughput structural biology 22 . Fragment methods have become a staple of modern drug discovery $^{23}$, using small collections $(100 \mathrm{~s}$ or $1000 \mathrm{~s}$ ) of small compounds ( $<300 \mathrm{Da})$ that bind promiscuously and thus sample a far larger chemical space than is achieved by HTS. The challenge is that the very weak binding of fragment hits necessitates highly sensitive biophysical detection, careful confirmation of binding and specialised medicinal chemistry expertise to advance hits to potency. Their promise is that potency can be achieved with high efficiency, simplifying the progression of molecules to biological or clinical impact.

While the screening experiment itself has long relied on the high throughput of solution methods like NMR or $\mathrm{SPR}^{23}$, rapid advances in technology and automation at synchrotron radiation sources $^{24}$ has made screening directly in crystal structures routinely possible at facilities like the XChem platform at Diamond Light Source ${ }^{25-28}$. These have been further enhanced by techniques such as mass spectrometry for the discovery of covalently binding fragments ${ }^{29}$.

In the current study, we screened Mpro of SARS-CoV-2 with over 1250 unique fragments, identifying 74 high-value fragment hits, including 23 non-covalent and 48 covalent hits in the active site, and 3 hits at the vital dimerization interface. Here, these data are detailed along with potential ways forward for rapid follow-up design of improved, more potent, compounds.

\section{Results}

Mpro crystallizes in a ligand-free form that diffracts to nearatomic resolution. We report the apo structure of SARS-CoV-2 Mpro with data to $1.25 \AA$. The construct we crystallised has native residues at both $\mathrm{N}$ - and C--terminals, without cloning truncations or appendages which could otherwise interfere with fragment binding. Electron density is present for all residues, including 26 alternate conformations, many of which were absent in previous lower resolution crystal structures. The protein crystallised with a single protein polypeptide in the asymmetric unit, and the catalytic dimer is provided by a symmetry-related molecule. The structure aligns closely with the Mpro structures from SARS-CoV-1 and MERS (rmsd of $0.52 \AA$ and $0.97 \AA$ respectively). The active site is sandwiched between two $\beta$-barrel domains, I (residue 10-99) and II (residue 100-182) (Fig. 1a). Domain III (residue 198-306), forms a bundle of alpha helices and is proposed to regulate dimerization ${ }^{30}$. The C-terminal residues, Cys300-Gln306, wrap against Domain II. However, the $\mathrm{C}$ terminal displays a degree of flexibility and wraps around domain III in the N3 inhibitor complex ${ }^{30}$ (PDB ID 6LU7
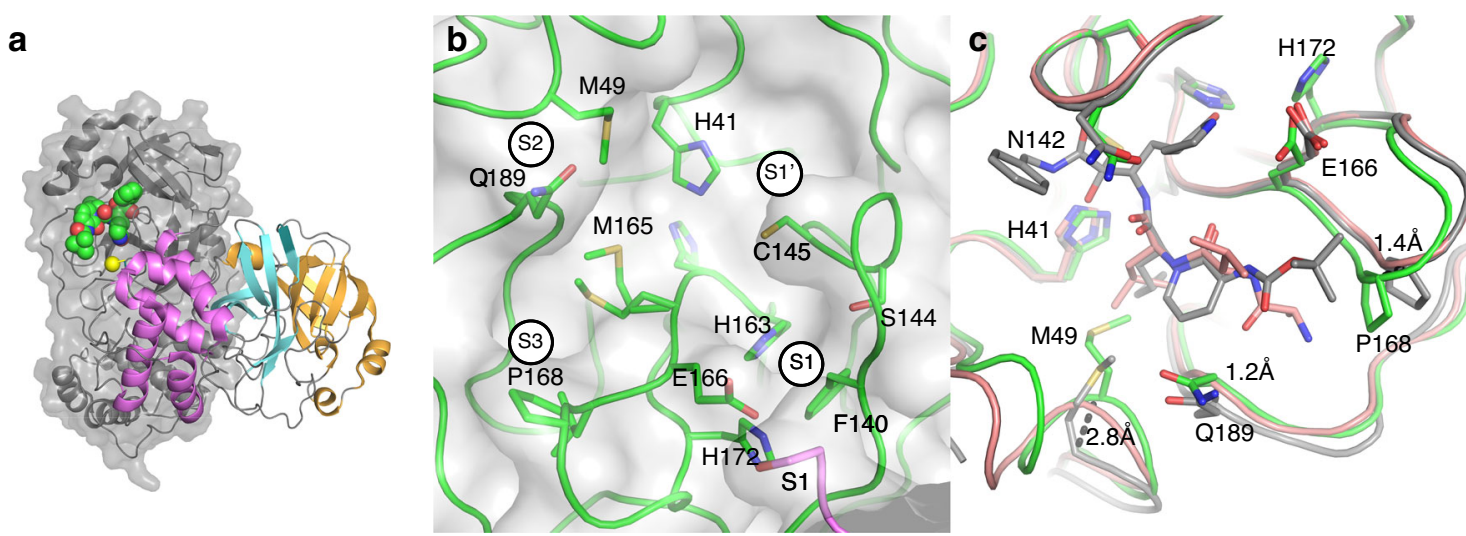

Fig. 1 The crystal structure of ligand free $\mathbf{M}^{\text {pro }}$ is amenable to X-ray fragment screening. a Cartoon representation of the $M$ pro dimer. The nearmost monomer is shown with secondary structure features coloured to demarcate domains I, II, and III, in orange, cyan, and violet respectively. The active site of the rear monomer is indicated by the presence of a peptide-based inhibitor in green, generated by aligning the ligand-free structure with pdb 6 Y $2 \mathrm{~F}$ [10.2210/pdb6y2f/pdb]. A yellow sphere indicates Ser1 from the dimer partner that completes the active site. $\mathbf{b}$ Residues of the active site are labelled, and subsites involved in ligand binding are shown with circles. c Active site plasticity is observed when comparing the apo structure to peptide inhibitor bound structures (green-Apo, grey-6Y2F [10.2210/pdb6y2f/pdb], pink 6LU7 [10.2210/pdb6lu7/pdb]). Displacement distances associated with loop movements are indicated. 


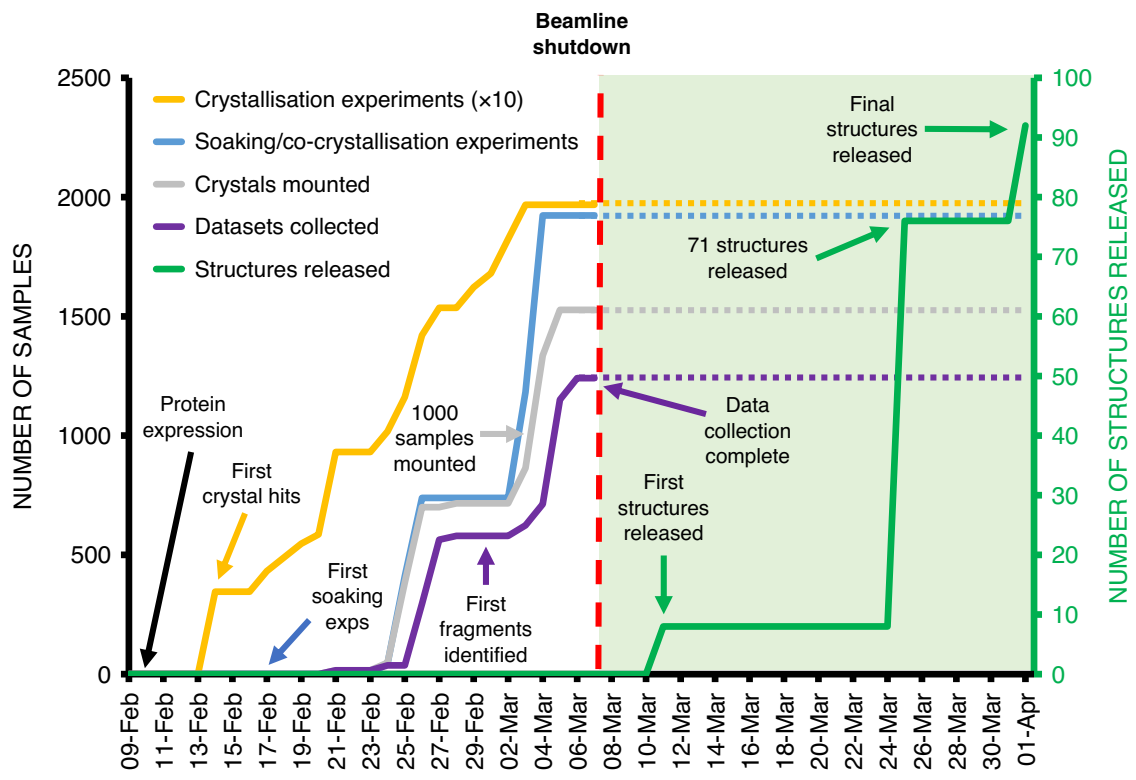

Fig. 2 Timeline of crystallographic fragment screen. Progress of the Mpro fragment screening experiment from the start of protein production and purification (9 Feb 2020) to the deposition and release of the high-resolution ligand-free structure of Mpro PDB ID 6YB7[10.2210/pdb6yb7/pdb] and the structures of the 96 fragment hits identified in the fragment screening campaign using the XChem platform at Diamond Light Source.

[https://doi.org/10.2210/pdb6lu7/pdb]). His41 and Cys145 comprise the catalytic dyad and dimerisation completes the active site by bringing Serl of the second dimer protomer into proximity with Glu166 (Fig. 1b). This aids formation of the substrate specificity pocket and the oxyanion hole ${ }^{10}$. Subsites have previously been identified in the active site based on interactions with peptide-based inhibitors and are shown in Fig. $1 b^{19,31}$. Comparisons with peptidebased inhibitor complexes ${ }^{19,31}$ suggest a degree of active site plasticity. In particular, the C-alphas of Met49, Pro168, Gln189 respectively show movements of $2.8 \AA, 1.4 \AA$, and $1.2 \AA$ in comparison to the a-ketoamide inhibitor bound Mpro structure $^{19}$ (PDB ID 6y2f [https://doi.org/10.2210/pdb6y2f/pdb], Fig. 1c).

The crystal form is well-suited for crystallographic fragment screening: although the percentage of solvent $(\sim 20 \%)$ is very low for a protein crystal, nevertheless clear channels are present that allow access to the active site through diffusion. Moreover, the tight packing and strong innate diffraction mean crystals are resistant to lattice disruption and degradation of diffraction by DMSO solvent when adding solubilised fragments to the crystallization drop.

Combined MS and crystallographic fragment screens reveal new binders of Mpro. Cysteine proteases are attractive targets for covalent inhibitors, and screening covalent fragments is known to be useful at identifying effective starting points ${ }^{32-36}$. To identify covalent starting points, we screened our previously described library of $\sim 1000$ mild electrophilic fragments ${ }^{29}$ against Mpro using intact protein mass spectrometry. Standard conditions of $200 \mu \mathrm{M}$ per electrophile for $24 \mathrm{~h}$ at $4{ }^{\circ} \mathrm{C}$ did not allow discrimination between hits. Screening at more stringent conditions $\left(5 \mu \mathrm{M}\right.$ per electrophile; $1.5 \mathrm{~h} ; 25^{\circ} \mathrm{C}$ ) resulted in $8.5 \%$ of the library labelling above $30 \%$ of protein (Supplementary Data 1). These hits revealed common motifs, and we focused on compounds that offer promising starting points.

Compounds containing $N$-chloroacetyl- $N^{\prime}$-sulfonamidopiperazine or $N$-chloroacetylaniline motifs were frequent hitters. Such compounds can be highly reactive. Therefore, we chose series members with relatively low reactivity for follow-up crystallization attempts. For another series of hit compounds, containing a $\mathrm{N}$-chloroacetyl piperidinyl-4-carboxamide motif
(Supplementary Data 1) which displays lower reactivity and were not frequent hitters in previous screens, we attempted crystallization despite their absence of labelling in the stringent conditions.

While mild electrophilic fragments are ideal for probing the binding properties around the active site cysteine, their small size prevents extensive exploration of the substrate-binding pocket. We performed an additional crystallographic fragment screen to exhaustively probe the Mpro active site, and to find opportunities for fragment merging or growing. The 68 electrophile fragment hits were added to crystals along with a total of 1176 unique fragments from 7 libraries (Supplementary Table 1). Noncovalent fragments were soaked ${ }^{26}$, whereas electrophile fragments were both soaked and co-crystallized as previously described ${ }^{29}$, to ensure that as many of the mass-spectrometry hits as possible were structurally observed. A total of 1742 soaking and 1139 cocrystallization experiments resulted in 1877 mounted crystals. While some fragments either destroyed the crystals or their diffraction, 1638 datasets with a resolution better than $2.8 \AA$ were collected. The best crystals diffracted to better than $1.4 \AA$, but diffraction to $1.8 \AA$ was more typical, and no datasets worse than $2.8 \AA$ were included in analysis (Supplementary Fig. 2). We identified 96 fragment hits using the PanDDA method ${ }^{37}$, all of which were deposited in the Protein Data Bank (Supplementary Data 2), but also immediately released through the Diamond Light Source website (https://www.diamond.ac.uk/covid-19. html), along with all protocols and experimental details. A timeline of experiments is shown in Fig. 2.

Non-covalent fragment hits reveal multiple targetable sub-sites in the active site. This unusually large screen identified 23 structurally diverse fragments that bind non-covalently and extensively sample features of the Mpro active site and its specificity pockets/subsites (Fig. 1), along with three hits exploring the dimer interface.

Eight fragments were identified that bind in the S1 subsite and frequently form interactions with the side chains of the key residues His163, through a pyridine ring or similar nitrogencontaining heterocycle, and Glu166 through a carbonyl group in an amide or urea moiety (Fig. 3). Several also reach across into 

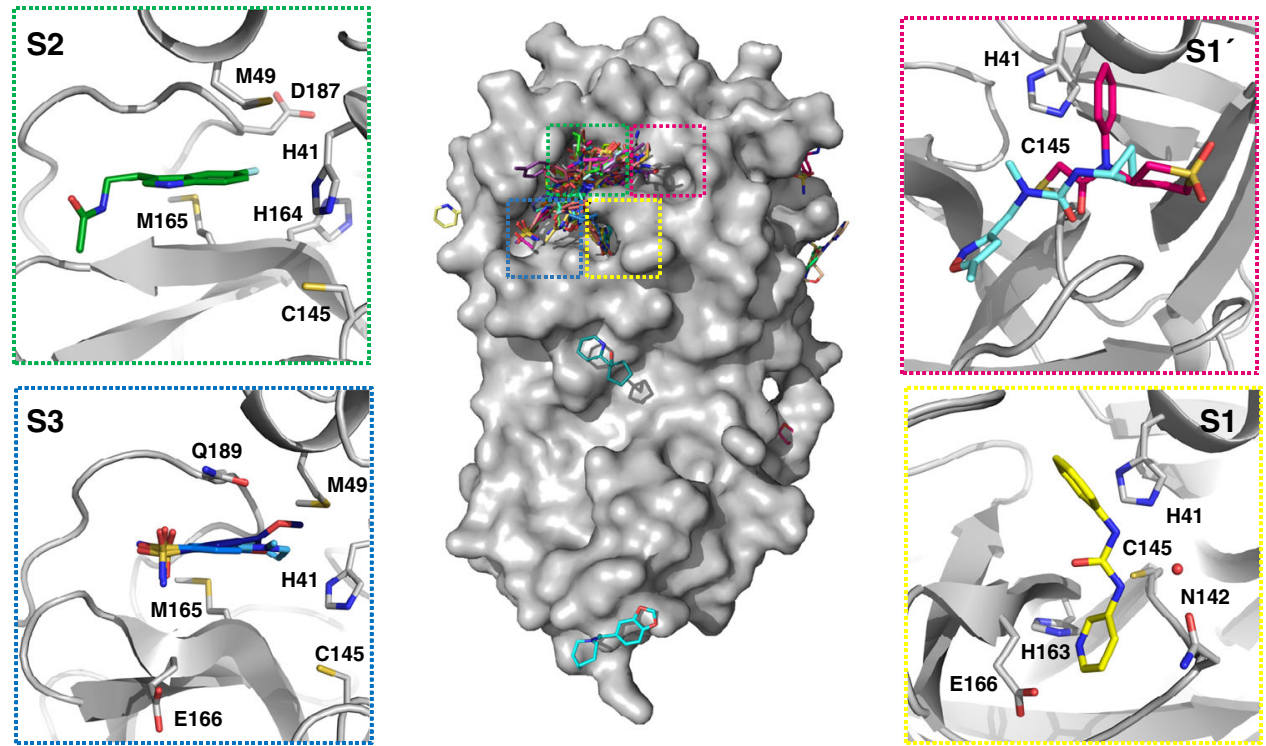

Fig. 3 Bound fragments sample the active site comprehensively. The central surface representation is of the Mpro monomer with all fragment hits shown as sticks, and active site subsites highlighted by coloured boxes. Each subsite is expanded along with a selection of hits to demonstrate common features and interactions. S1: Z44592329 (x0434); S1': Z369936976 (×0397) in aquamarine and PCM-0102372 (×1311) in magenta bound to active site cysteine; S2: Z1220452176 (x0104); S3: Overlay of Z18197050 (×0161), Z1367324110 (×0195) and NCL-00023830 (×0946).
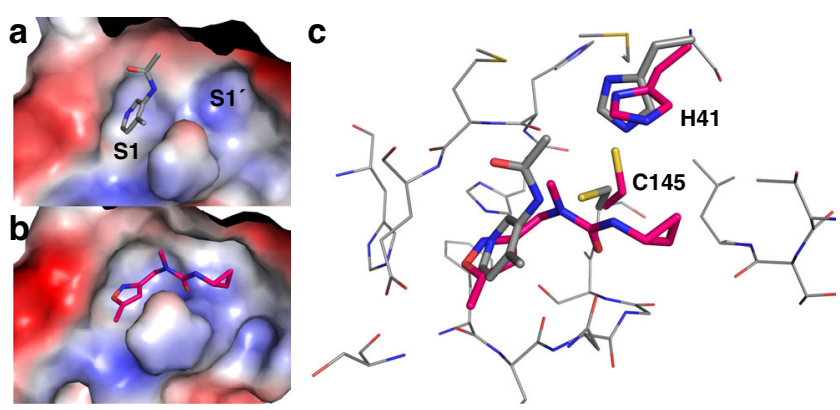

Fig. 4 Plasticity of $\mathrm{S1}^{\prime}$ is revealed by fragment Z369936976 $(\times 0397)$. Comparing the electrostatic surfaces of Z1129283193 (×0107) a The most commonly observed conformation, with that of Z369936976 (x0397). b How the shape of S1 and S1' can change. c Sidechain movement of catalytic residues Cys145 and His41 upon binding of Z369936976 (×0397, magenta) compared to Z1129283193 (×0197, grey).

the S2 subsite. Subsite S2 has previously demonstrated greater flexibility in comparison to the other subsites, adapting to smaller substituents in peptide-based inhibitors but with a preference for leucine or other hydrophobic residues ${ }^{19}$. Many fragments bound at this location, which we termed the "aromatic wheel" because of a consistent motif of an aromatic ring forming hydrophobic interactions with Met 49 or $\pi-\pi$ stacking with His41, with groups variously placed in 4 axial directions. Particularly notable is the vector into the small pocket between His164, Met165 and Asp187, exploited by three of the fragments (Z1220452176 (x0104), Z219104216 (x0305) and Z509756472 (×1249)) with fluoro and cyano substituents (Fig. 3).

Of the four fragments exploring subsite S3, three contain an aromatic ring with a sulfonamide group forming hydrogen bonds with Gln189 and pointing out of the active site towards the solvent interface (Fig. 3). These hits have expansion vectors suitable for exploiting the same His164/Met165/Asp187 pocket mentioned above.

The experiment revealed one notable conformational variation, which was exploited by one fragment only (Z369936976 $(\times 0397)$;
Fig. 4): a change in the sidechains of the key catalytic residues His41, Cys145 alters the size and shape of subsite $S 1^{\prime}$ and thus the link to subsite S1. This allows the fragment to bind, uniquely, to both $\mathrm{S} 1$ and $\mathrm{S}^{\prime}$. In $\mathrm{S} 1$, the isoxazole nitrogen hydrogen-bonds to His163, an interaction that features in several other hits; and in $S 1^{\prime}$, the cyclopropyl group occupies the region sampled by the covalent fragments. Notably, the $\mathrm{N}$-methyl group offers a vector to access the S2 and S3 subsites.

It is established that the biological unit for similar viral proteases such as the SARS-CoV-1 protease is a dimer ${ }^{38}$, and that mutations at the dimer interface can disrupt proteases activity 39,40 even at long range ${ }^{41}$. Thus, compounds that interfere with dimerization might serve as quasi-allosteric inhibitors of protease activity. In this study three compounds bound at accessible sites of the dimer interface, that conceivably could be exploited to design compounds to disrupt the Mpro dimer.

Fragment Z1849009686 ( $\times 1086$; Fig. 5a) binds in a hydrophobic pocket formed by the sidechains of Met6, Phe8, Arg298 and Val303. It also mediates two hydrogen bonds to the sidechain of Gln127 and the backbone of Met6. Its binding site is $<7 \AA$ away from Ser139, whose mutation to alanine in SARS-CoV-1 protease reduced both dimerization and protease activity by about $50 \% \%^{39,42}$. Z264347221 ( $\times 1187$, Fig. 5b) binds similarly in a hydrophobic pocket made by Met6, Phe 8 and Arg298 in one of the protomers, extending across the dimer interface to interact with Ser123, Tyr118 and Leu141 of the second protomer, including hydrogen bonds with the sidechain and backbone of Ser123. Finally, POB0073 (x0887; York 3D library; Fig. 5c), binds only $4 \AA$ from Gly2 at the dimer interface and is encased between Lys137 and Val171 of one protomer and Gly2, Arg4, Phe3, Lys5 and Leu282 of the second, including two hydrogen bonds with the backbone of Phe3.

Covalent fragment hits reveal several tractable series. The screen further yielded 48 structures of fragments covalently bound to the nucleophilic active site Cys145, and substrate subsite S1'. The majority (44) fall into series explored in the massspectrometry experiment and the remainder came from other libraries. 

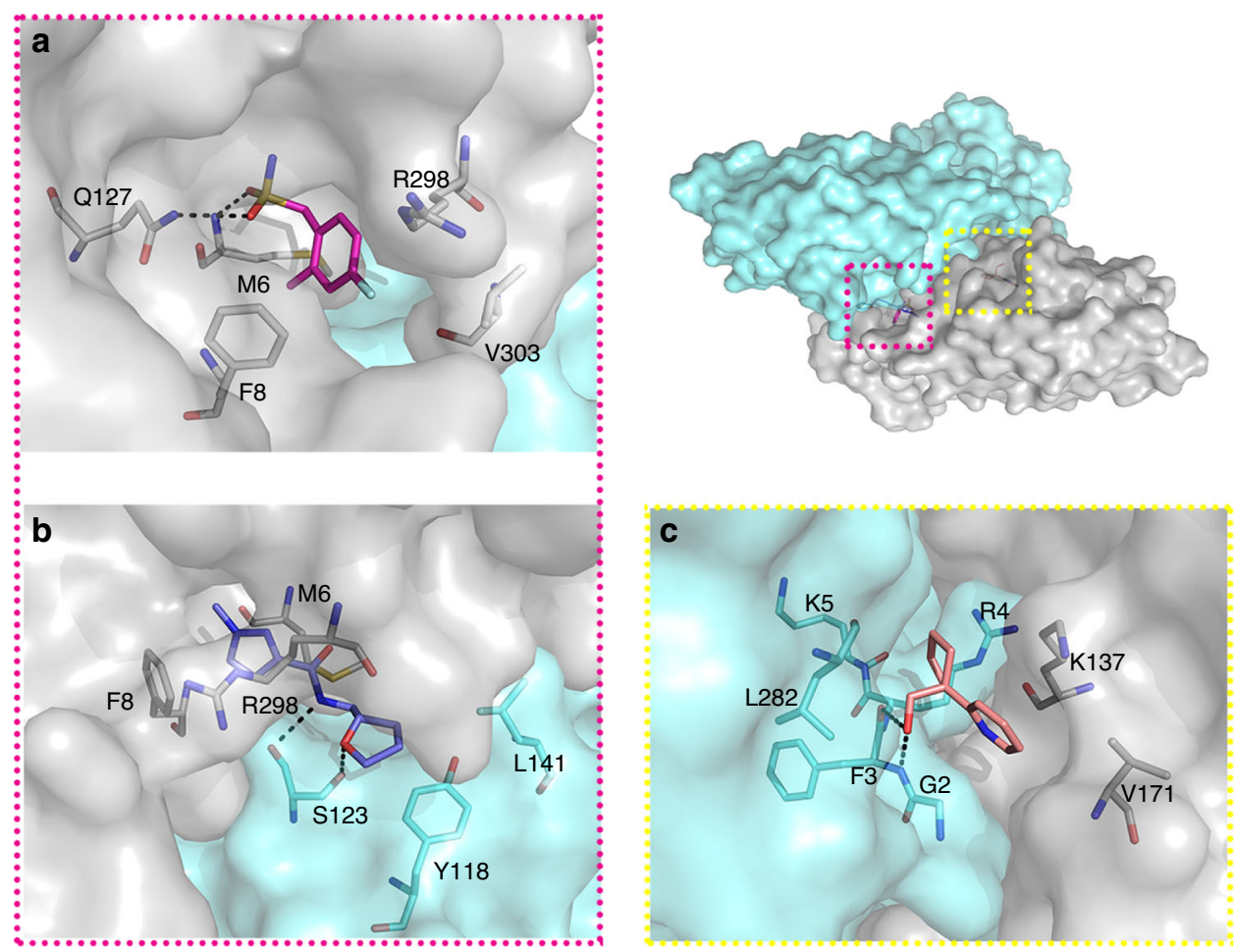

Fig. 5 Fragments at dimer interface indicate opportunities for allosteric modulation. The overview shows the surface of the Mpro dimer, the protomers in grey and cyan. Fragments and surrounding residues are shown as sticks and hydrogen bonds in dashed black lines. a Z1849009686 ( $\times 1086)$. b Z264347221 (×1187). c POB0073 (×0887)

In all structures with bound electrophiles, the $N$-chloroacetyl carbonyl oxygen atom forms either two or three hydrogen bonds with the backbone amide hydrogens of Gly143, Ser144 or Cys145 (Fig. 6a-c). All three compounds containing the $\mathrm{N}$-chloroacetyl piperidinyl-4-carboxamide motif (Fig. 6a) adopt a similar binding mode pointing towards the S2 pocket, and one (PCM-0102389, $\times 1358)$ is able to form an additional hydrogen bond with the side chain of Asn142.

Compounds with the $N$-chloroacetyl- $N$ '-sulfonamido-piperazine motif (Fig. 6b) adopt a bent shape, pointing towards the S2 pocket where appropriate space-filling substituents are attached to the phenyl moiety (PCM-0102353 $(\times 1336)$ and PCM-0102395 $(\times 0774))$; otherwise, they point towards the solvent. Most of the latter 8 structures feature a halophenyl moiety which resides closely to Asn142, hinting at weak halogen-mediated interactions ${ }^{43}$.

Eight compounds with a $N$-chloroacetyl- $N^{\prime}$-carboxamidoand $N$-chloroacetyl- $N^{\prime}$-heterobenzyl-piperazine motif crystallized in one binding mode with respect to the piperazinyl moiety (Fig. 6c) (with one exception, PCM-0102287 (×0830)). Two structures (PCM-0102277 (×1334), PCM-0102169 $(\times 1385))$ with a 5-halothiophen-2-ylmethylene moiety exploit lipophilic parts of S2, which is also recapitulated by the thiophenyl moiety in an analogous carboxamide (PCM-0102306 (x1412)). The other five structures point mainly to S2, offering an accessible growth vector towards the nearby S3 pocket.

A series of compounds containing a N-chloroacetyl piperidinyl-4-carboxamide motif showed promising binding modes. To follow-up on these compounds, we performed rapid second-generation compound synthesis. Derivatives of this chemotype were accessible in milligram-scale by the reaction of $\mathrm{N}$-chloroacetyl piperidine-4-carbonyl chloride with various inhouse amines, preferably carrying a chromophore to ease purification. These new compounds were tested by intact protein mass-spectrometry to assess protein labelling ( $5 \mu \mathrm{M}$ compound; $1.5 \mathrm{~h}$ incubation, RT; Supplementary Data 3). Amides derived from non-polar amines mostly outcompeted their polar counterparts, hinting at a targetable lipophilic sub-region in this direction. The two amides with the highest labelling PG-COV35 and PG-COV-34 (Fig. 6g, h) highlight the potential for further synthetic derivatization by amide $\mathrm{N}$-alkylation or cross-coupling, respectively.

The screen revealed unexpected covalent warheads from the series of 3-bromoprop-2-yn-1-yl amides of N-acylamino acids. Colloquially termed PepLites, this library was developed to map non-covalent interactions of amino acid sidechains in proteinprotein interaction hotspots, with the acetylene bromine intended, as for FragLites ${ }^{44,45}$, as a detection tag by anomalous dispersion in X-ray crystallography. However, bromoalkynes can also act as covalent traps for activated cysteine thiols ${ }^{46}$ (Fig. 6d).

Two PepLites, containing threonine (NCL-00025058 $(\times 0978))$ and asparagine (NCL00025412 $(\times 0981)$ ) bound covalently to the active site cysteine (Cys145), forming a thioenolether via C-2 addition with loss of bromine (Fig. 6e, f). The covalent linkage was unexpected and evidently the result of significant noncovalent interactions, specific to these two PepLites, that position the electrophile group for nucleophilic attack. We note the sidechains make hydrogen-bonding interactions with various backbone $\mathrm{NH}$ and $\mathrm{O}$ atoms of Thr26 and Thr24; in the case of threonine, it was the minor $2 \mathrm{R}, 3 \mathrm{R}$ diastereomer (corresponding to D-allothreonine) that bound. The only other PepLite observed (tyrosine, NCL-00024905 (×0967)) bound non-covalently to a different subsite.

The highlighted structure-activity relationships are important for further optimisation. Bromoalkynes have intrinsic thiol reactivity that is lower than that of established acrylamidebased covalent inhibitors ${ }^{46}$, which is in general desirable. The geometry of the alkyne and its binding mode also suggest that it 
a

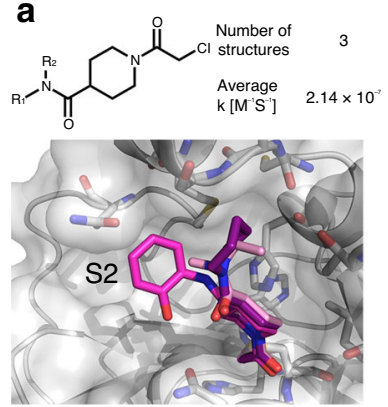

b

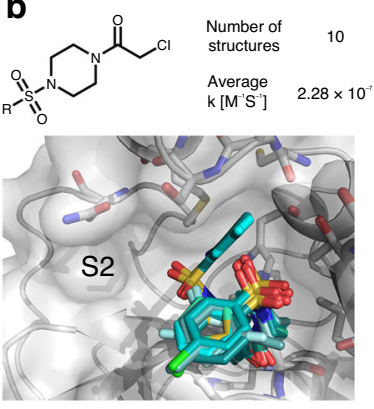

C

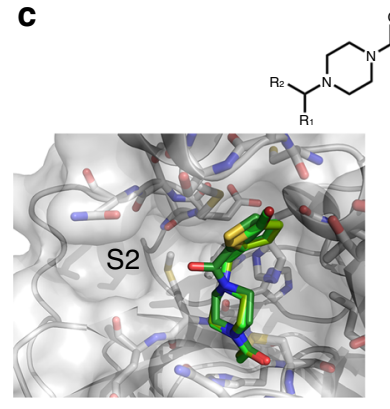

d

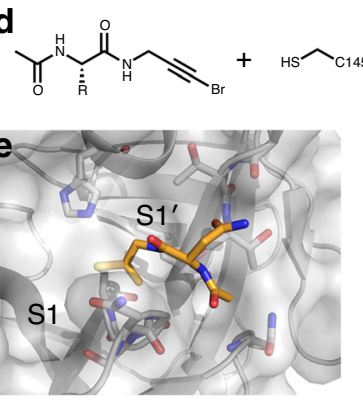

g
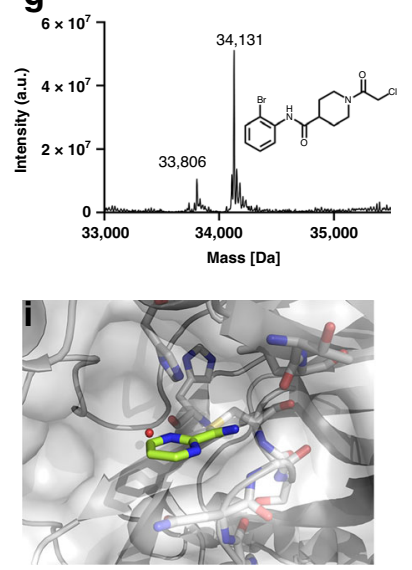
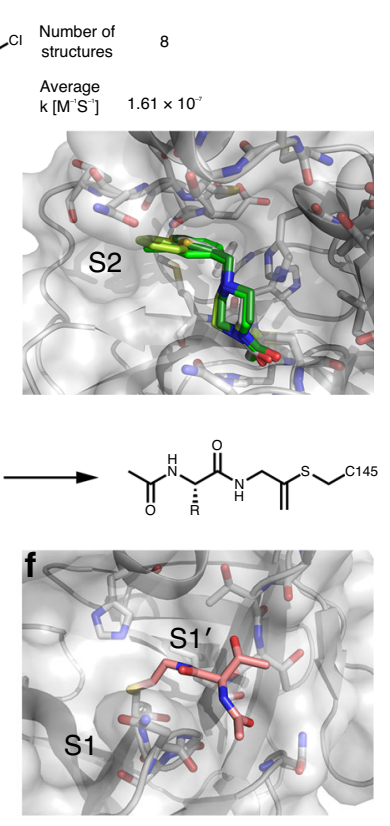

h
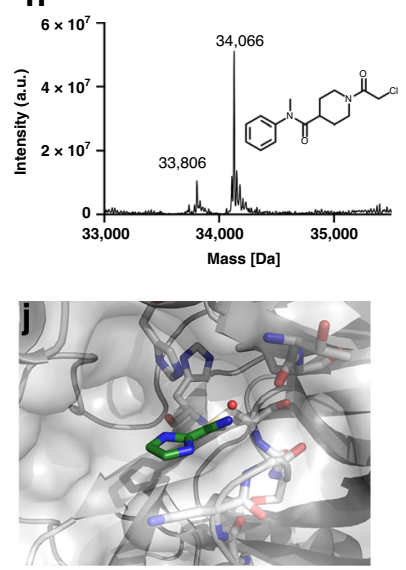

could be replaced by reversible covalent groups such as nitriles, which would be guided by the same non-covalent interactions but are better established as cysteine protease inhibitors.

Two covalent hits (2-cyanopyrimidine (Cov_HetLib 030 $(\times 2097)$ and 2-cyanoimidazole (Cov_HetLib $053 \quad(\times 2119)$ came from a library of small heterocyclic electrophiles ${ }^{47}$. These are essentially covalent MiniFrags ${ }^{48}$, comprising five and sixmembered nitrogen-containing heterocycles with electronwithdrawing character that activates small electrophilic substituents (halogens, ethylyl, vinyl and nitrile groups).

Both hits bound to Cys145 through an imine (Fig. 6i, j), positioned by a local hydrogen bond network involving imine and

Fig. 6 Covalent fragments are anchored at Cys145 and sample different regions of the orthosteric $\mathbf{M}^{\text {pro }}$ binding pocket. a Fragments containing $\mathrm{N}$-chloroacetyl piperidinyl-4-carboxamide motif. b Fragments containing $\mathrm{N}$ chloroacetyl- $\mathrm{N}^{\prime}$-sulfonamido-piperazine motif. c Fragments containing $\mathrm{N}$ chloroacetyl- $N^{\prime}$-carboxamido- and $N$-chloroacetyl- $N^{\prime}$-heterobenzylpiperazine in two binding modes. The second order kinetic constants refer to the intrinsic thiol reactivity of these fragment hits as previously measured ${ }^{29}$. d Reaction schema of the unexpected covalent modification to Cys145 by PepLites hits. e Threonine PepLite (NCL-00025058 (x0978)) bound covalently to active site cysteine. $\mathbf{f}$ Asparagine PepLite (NCL00025412 (x0981)) bound to active site cysteine. Labelling of $M^{\text {pro }}$ by 2nd generation compounds proven by intact protein LC-MS: $\mathbf{g}$ Labelling by PGCOV-35; $\mathbf{h}$ Labelling by PG-COV-34. Covalently bound cyclic electrophiles: i Cov_HetLib $030(\times 2097)$ and j Cov_HetLib $053(\times 2119)$.

heterocyclic $\mathrm{N}$ atoms. One of these free amines provides an immediate growth vector towards the catalytic pocket. The compounds have reasonable stability in water ${ }^{49}$ and limited reactivity against GSH ( $t_{1 / 2}=2.2$ and $52.3 \mathrm{~h}$, respectively), well above suggested reactivity limits ${ }^{50}$. They are also inactive against various covalent targets (HDAC8, MAO-A, MAO-B, MurA) and benchmark proteins.

\section{Discussion}

The data presented herein provide many clear routes to developing potent inhibitors of Mpro from SARS-CoV-2. The bound fragments comprehensively sample all subsites of the active site, revealing diverse expansion vectors, and the electrophiles provide extensive data, systematic as well as serendipitous, for designing covalent compounds.

It is widely accepted that new small-molecule drugs cannot be developed fast enough to help against COVID-19. Nevertheless, as the pandemic threatens to remain a long-term problem and vaccine candidates do not promise complete and lasting protection, antiviral molecules will remain an important line of defence. Such compounds will also be needed to fight future pandemics ${ }^{10}$. Our data will accelerate such efforts: therapeutically, through the design of new molecules and to inform ongoing efforts at repurposing existing drugs; and for research, through the development of probe molecules ${ }^{51}$ to understand viral biology. One example is the observation that fragment $\mathrm{Z} 1220452176(\times 0104)$ is a close analogue of melatonin, although in this case, it is unlikely that melatonin mediates direct antiviral activity through inhibition of Mpro, given its low molecular weight; nevertheless, melatonin is currently in clinical trials to assess its immune-regulatory effects on COVID19 (Clinicaltrials. gov identifier NCT04353128).

In line with the urgency, results were made available online immediately for download. In addition, since exploring $3 \mathrm{D}$ data requires specialised tools ${ }^{52,53}$, hits were made accessible on the Fragalysis webtool (https://fragalysis.diamond.ac.uk) that allows easy exploration of the hits in interactive $3 \mathrm{D}$.

All released models were stringently assessed for reliability. On the one hand, the whole data analysis process necessarily relied heavily on automation that, since its initial testing, has been extensively validated on over 100 experiments at the XChem facility, indicating the processes are robust in generating highquality atomic models. On the other hand, the final selection of models was by subjective evaluation of the fit of each atomic model to electron density. All models were therefore reviewed by multiple authors prior to release, and a subjective confidence assigned to each (Supplementary Data 2). The evidence used was the unbiased event density generated by the PanDDA method ${ }^{37}$, which uses multi-dataset averaging to extract signal from electron 

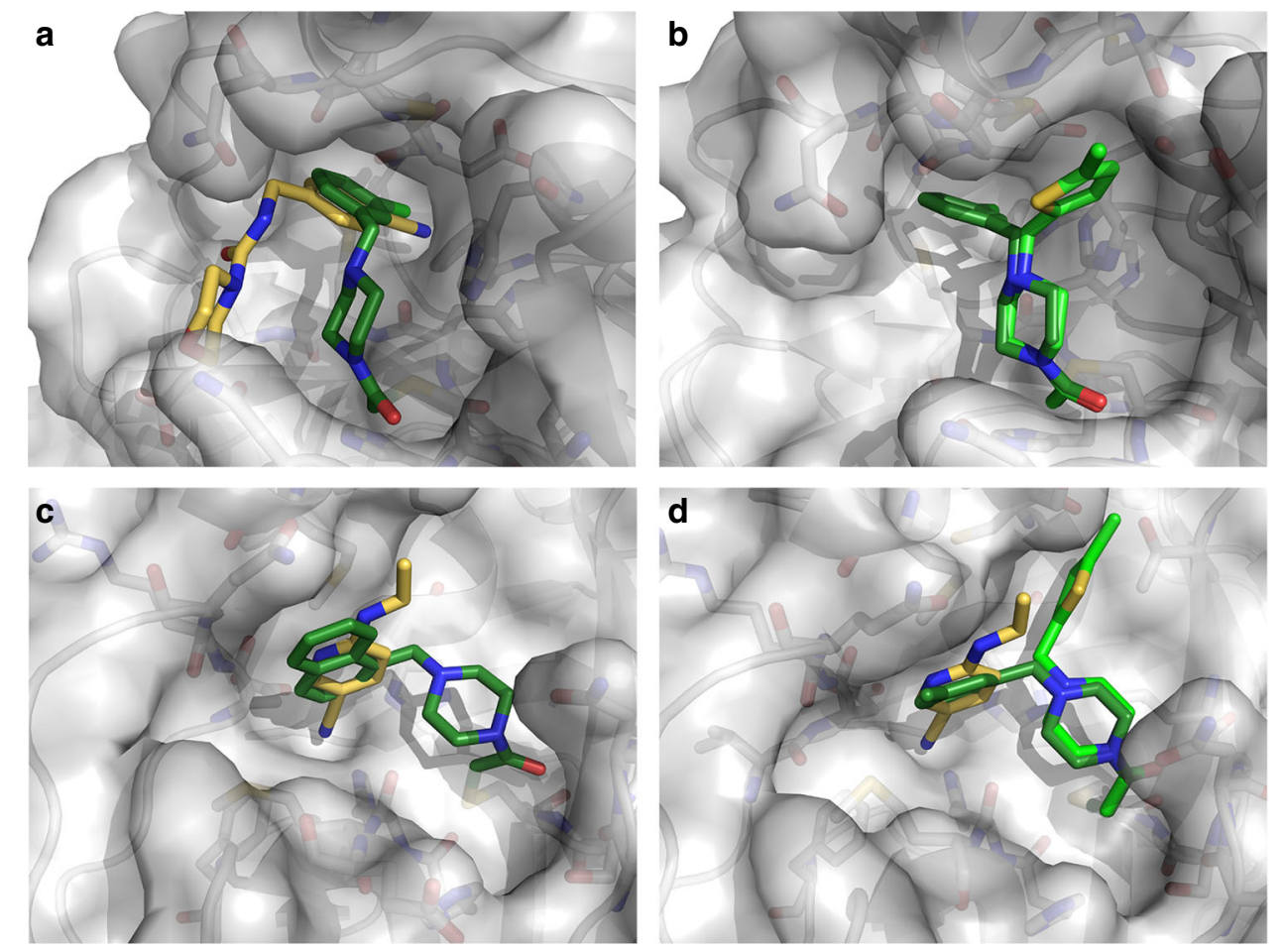

Fig. 7 Fragment merging opportunities can be directly inferred from many hits. Covalently bound fragments are in green shades, and non-covalent fragments in yellow. a Overlay of Z509756472/×1249 and PCM-0102269/×0770. b Overlay of PCM-0102277/×1334 and PCM-0102269/×0770. c Overlay of PCM-0102287/×0830 and Z219104216/×0305. d Overlay of PCM-0102340/×0692, PCM-0102277/×1334 and Z219104216/×0305.

density that would historically have been considered too noisy to be convincingly interpretable ${ }^{54}$; accordingly, even ligands with low occupancy $(<40 \%)$ could be confidently assessed (Supplementary Note 1$)$. Likewise this means that poor diffraction is a common occurrence due to the crystal handling steps required for soaking of fragments. However, for each dataset, the dominant source of noise is low occupancy and not phase bias, since crystals and thus datasets are only subtly different (Supplementary Note 1).

We have previously demonstrated the benefits of merging covalent and non-covalent fragments to make dramatic improvements in potency ${ }^{29}$. Our dataset offers numerous opportunities and some conservative examples are shown in Fig. 7. These can be expected to result in potent $M^{p r o}$ binders and compound synthesis is ongoing.

Collectively, the covalent hits provide rational routes to inhibitors of low reactivity and high selectivity. Rationally designed covalent drugs are gaining traction, with many recent FDA approvals ${ }^{55,56}$. Their design is based on very potent non-covalent binding, that allows precise orientation of a low reactivity electrophile, so that formation of the covalent bond is reliant on binding site specificity, with minimal off-target effects ${ }^{57-59}$. For fear of over-reactivity, covalent inhibitors are expunged from high-throughput screening libraries and are typically considered as PAINS compounds ${ }^{60-62}$. The challenge of tuning reactivity, and the danger of reactivity-based artefacts, are considered to be particularly marked for highly reactive nucleophiles such as the catalytic cysteine of many proteases. This is evidenced by the very high hit-rate we saw in our preliminary screen of electrophiles in which more than 150 fragments labelled $\mathrm{M}^{\text {pro }}$ by $>50 \%$. Robust characterization of the fragments' reactivity ${ }^{29}$, and continuous evaluation of general thiol reactivity in the selection of lead series and during hit-to-lead optimization can address this challenge. We note here that most of the electrophiles that we screened (chloroacetamides and acrylamides) form irreversible adducts with the target cysteine, whereas many protease inhibitors contain aldehydes, nitriles and $\alpha$-ketoamides, that can form reversible covalent bonds.

The scale of this experiment, particularly the diversity of libraries and density of results, likely sets a new benchmark for ensuring a crystal-based fragment screen accelerates progression of hits. Even cursory inspection of the fragment structures indicates a very large "merge space", i.e., the collection of compounds that can be designed directly from spatial juxtapositions of fragments. Such merges, which can be made to populate all four subsites, might achieve potency synergistically, because the observed interactions can be assumed to be in near-optimal configurations, given how few there are per fragment. A thorough exploration of merge space might be best achieved formulaically, using computational workflows that additionally filter undesirable molecular properties, assess synthetic tractability and predict binding affinity. However, such integrated approaches are not currently available in the public domain. We hope this dataset will help spur their development and testing.

Another promising effort to explore the potential of this premise is the COVID Moonshot project (https://covid.postera.ai/ covid), where a selection of merges will be experimentally tested, with data promptly made public. We trust that this resource will enable the development of many new tools, approaches and ultimately viable treatment candidates for COVID19.

\section{Methods}

Protein expression. The expression vector was constructed with a codonoptimised gene fragment, synthesized by Integrated DNA Technologies, which included in-fusion compatible ends for direct insertion into BamHI-XhoI digested pGEX-6P-1 (Supplementary Table 2). The resulting plasmid yields native $\mathrm{N}$ - and $\mathrm{C}$-termini upon $3 \mathrm{C}$ protease treatment during the purification. Multiple transformant colonies were used to inoculate a starter culture supplemented with $100 \mu \mathrm{g} /$ $\mathrm{ml}$ Carbenicillin. The culture was then grown to log phase for $\sim 8 \mathrm{~h}$. Ten millilitres of the starter culture was used to inoculate one litre of auto induction medium supplemented with $10 \mathrm{ml}$ of glycerol and $100 \mu \mathrm{g} / \mathrm{ml}$ carbenicillin. The cultures were 
grown at $37^{\circ} \mathrm{C}, 200 \mathrm{rpm}$ for $5 \mathrm{~h}$ then switched to $18^{\circ} \mathrm{C}, 200 \mathrm{rpm}$ for $10 \mathrm{~h}$. The cells were harvested by centrifugation and stored at $-80^{\circ} \mathrm{C}$

Protein purification. Cells were resuspended in $50 \mathrm{mM}$ Tris $\mathrm{pH} 8,300 \mathrm{mM} \mathrm{NaCl}$, $10 \mathrm{mM}$ Imidazole, $0.03 \mu \mathrm{g} / \mathrm{ml}$ Benzonase. The cells were disrupted on a highpressure homogeniser ( 3 passes, $30 \mathrm{kpsi}, 4^{\circ} \mathrm{C}$ ). The lysate was clarified by centrifugation at $50,000 \times g$. The supernatant was then applied to a Nickel-NTA gravity column and washed and eluted with $50 \mathrm{mM}$ Tris $\mathrm{pH} 8,300 \mathrm{mM} \mathrm{NaCl}$, and 25-500 mM imidazole pH 8. N-terminal His-tagged HRV 3 C Protease was then added to the eluted protein at 1:10 w/w ratio. The mixture was then dialysed overnight at $4{ }^{\circ} \mathrm{C}$ against $50 \mathrm{mM}$ Tris $\mathrm{pH} 8,300 \mathrm{mM} \mathrm{NaCl}, 1 \mathrm{mM}$ TCEP. The following day, the HRV $3 \mathrm{C}$ protease and other impurities were removed from the cleaved target protein by reverse Nickel-NTA. The relevant fractions were concentrated and applied to an S200 16/60 gel filtration column equilibrated in $20 \mathrm{mM}$ Hepes $\mathrm{pH} 7.5,50 \mathrm{mM} \mathrm{NaCl}$ buffer. The protein was concentrated to $30 \mathrm{mg} / \mathrm{ml}$ using a $10 \mathrm{kDa}$ MWCO centrifugal filter device.

Crystallisation and structure determination. Protein was thawed and diluted to $5 \mathrm{mg} / \mathrm{ml}$ using $20 \mathrm{mM}$ Hepes $\mathrm{pH} 7.5,50 \mathrm{mM} \mathrm{NaCl}$. The sample was centrifuged at $100,000 \times g$ for $15 \mathrm{~min}$. Initial hits were found in well F2 of the Proplex crystallisation screen, $0.2 \mathrm{M} \mathrm{LiCl}, 0.1 \mathrm{M}$ Tris $\mathrm{pH} 8,20 \%$ PEG $8 \mathrm{~K}$. These crystals were used to prepare a seed stock by crushing the proteins with a pipette tip, suspending in reservoir solution and vortexing for $60 \mathrm{~s}$ in the reservoir solution with $\sim 10$ glass beads ( $1.0 \mathrm{~mm}$ diameter, BioSpec products). Adding DMSO to the protein solution to a concentration of $5 \%$ and performing microseed matrix screening, many new crystallisation hits were discovered in commercial crystallisation screens. Following optimisation, the final crystallisation condition was 11\% PEG $4 \mathrm{~K}, 6 \%$ DMSO, 0.1 M MES pH 6.7 with a seed stock dilution of $1 / 640$. The seeds were prepared from crystals grown in the final crystallisation condition. The drop ratios were $0.15 \mu \mathrm{l}$ protein, $0.3 \mu \mathrm{l}$ reservoir solution, $0.05 \mu \mathrm{l}$ seed stock. Crystals were grown using the sitting drop vapour diffusion method at $20^{\circ} \mathrm{C}$ and appeared within $24 \mathrm{~h}$.

Initial diffraction data were collected on beamline I04 at Diamond Light Source on a crystal grown in $0.1 \mathrm{M}$ MES pH 6.5, 5\% PEG6K, cryoprotected using 30\% PEG400. Data were processed using Dials ${ }^{63}$ via Xia2 ${ }^{64}$. The dataset was phased with the SARS-CoV-2 Mpro in complex with the N3 inhibitor crystal structure (PDB:6LU7 [https://doi.org/10.2210/pdb6lu7/pdb]) using Molrep in CCP4i2. Further datasets were collected on I04-1 at Diamond Light Source on crystals grown using the $0.1 \mathrm{M}$ MES pH 6.5, 15\% PEG4K, 5\% DMSO condition. To create a high-resolution dataset, datasets from 7 crystals were scaled and merged using Aimless ${ }^{65}$. Crystal structures were manually rebuilt in $\operatorname{Coot}^{66}$ and refined using Refmac $^{67}$ and Buster ${ }^{68}$. This structure is deposited in the PDB under ID 6YB7 [https://doi.org/10.2210/pdb6yb7/pdb].

Electrophile fragment LC/MS screen. $2 \mu \mathrm{M}$ Mpro was incubated in $50 \mathrm{mM}$ Tris $\mathrm{pH} 8300 \mathrm{mM} \mathrm{NaCl}$ for $1.5 \mathrm{~h}$ at $25^{\circ} \mathrm{C}$. For initial electrophile fragment library screen, $30 \mu \mathrm{l}$ protein with pools of $4-5$ electrophile fragments, $7.5 \mathrm{~nL}$ each from 20 $\mathrm{mM}$ DMSO stocks and for other runs $50 \mu \mathrm{l}$ protein with $0.5 \mu \mathrm{l}$ compounds from $0.5 \mathrm{mM}$ DMSO stocks. The reaction was quenched by adding formic acid to $0.4 \%$ final concentration. The LC/MS runs were performed on a Waters ACUITY UPLC class $\mathrm{H}$ instrument, in positive ion mode using electrospray ionization. UPLC separation used a C4 column $(300 \AA, 1.7 \mu \mathrm{m}, 21 \mathrm{~mm} \times 100 \mathrm{~mm})$. The column was held at $40^{\circ} \mathrm{C}$ and the autosampler at $10^{\circ} \mathrm{C}$. Mobile solution A was $0.1 \%$ formic acid in water, and mobile phase B was $0.1 \%$ formic acid in acetonitrile. The run flow was $0.4 \mathrm{~mL} / \mathrm{min}$ with gradient $20 \%$ B for $4 \mathrm{~min}$, increasing linearly to $60 \%$ B for $2 \mathrm{~min}$, holding at $60 \% \mathrm{~B}$ for $0.5 \mathrm{~min}$, changing to $0 \% \mathrm{~B}$ in $0.5 \mathrm{~min}$, and holding at $0 \%$ for 1 min. The mass data were collected on a Waters SQD2 detector with an $\mathrm{m} / z$ range of $2-3071.98$ at a range of $1000-2000 \mathrm{~m} / \mathrm{z}$. Raw data were processed using openLYNX and deconvoluted using MaxEnt. For each well, deconvoluted peaks were searched to match either the unlabelled protein, or labelled protein with one or two of the compounds in the well. Labelling percentage for a compound was determined as the $\%$ of a specific compound adduct, divided by the overall detected protein species. Peaks whose mass could not be assigned were discarded from the overall labelling calculation. Wells are regarded as "bad wells" if their spectra appeared to be of a degraded protein (low intensity and deformed peak shape) or if after deconvolution there were no clear peaks (high noise levels). No labelling was assigned for bad wells.

Fragment screening. Fragments were soaked into crystals by adding dissolved compound directly to the crystallisation drops. The following libraries were screened: the DSipoised library (Enamine), a version of the poised library ${ }^{25}$; a version of the MiniFrags library ${ }^{48}$ assembled in-house; the FragLites library ${ }^{45}$; a library of shape-diverse 3D fragments ("York3D")69; heterocyclic electrophiles 47 and the SpotFinder library. All fragments were in 100\% DMSO at varying stock concentrations, detailed at https://www.diamond.ac.uk/Instruments/Mx/FragmentScreening/Fragment-Libraries.html). In brief, $55 \mathrm{nl}$ of fragment stock solutions in DMSO (DSI-poised, FragLite, PepLites, York 3D, Covalent Heterocylces and SpotFinder all at $500 \mathrm{mM}$, MiniFrags at $1 \mathrm{M}$ and Cysteine covalent library at 20 $\mathrm{mM}$ ) were transferred directly to $500 \mathrm{nl}$ crystallisation drops using an ECHO liquid handler giving a final compound concentration of $2-100 \mathrm{mM}$ and DMSO concentration of $10 \%$. Drops were incubated at room temperature for $\sim 1 \mathrm{~h}$ prior to mounting and flash cooling in liquid nitrogen without the addition of further cryoprotectant

Electrophile fragments identified by mass spectrometry were soaked by the same procedure as the other libraries, but in addition, they were also co-crystallised in the same crystallisation condition as for the apo structure. The protein was incubated with 10-20-fold excess compound (molar ratio) for $\sim 1 \mathrm{~h}$ prior to the addition of the seeds and reservoir solution (following Resnick et al. ${ }^{29}$ ).

Data were collected at the beamline I04-1 at $100 \mathrm{~K}$ and processed with the fully automated pipelines at Diamond ${ }^{63,70,71}$, which variously combine $\mathrm{XDS}^{72}$, xia2 ${ }^{64}$, autoPROC ${ }^{73}$ and DIALS ${ }^{63}$, and select resolution limits algorithmically; no manual curation of processing parameters was applied. Further analysis was performed through XChemExplorer ${ }^{27}$ : for each dataset, the version of processed data was selected by the default XChemExplorer score, and electron density maps were generated with Dimple ${ }^{74}$. Ligand-binding events were identified using PanDDA ${ }^{37}$ (both the released version 0.2 and a pre-release development version (https:// github.com/ConorFWild/pandda)), and ligands were modelled into PanDDAcalculated event maps using $\operatorname{Coot}^{66}$. Restraints were calculated with ACEDRG or GRADE $^{75,76}$, structures were refined with Refmac ${ }^{67}$ and Buster ${ }^{68}$, and models and quality annotations cross-reviewed. Further elaboration of the PanDDA analysis is provided in the Supplementary Note 1.

Coordinates, structure factors and PanDDA event maps for all data sets are deposited in the Protein Data Bank under group deposition ID G_1002135, G_1002151, G_1002152, G_1002153, G_1002156 and G_1002157. Data collection and refinement statistics are summarised in Supplementary Data 4. The groundstate structure and all corresponding datasets are deposited under PDB ID 5R8T [https://doi.org/10.2210/pdb5r8t/pdb].

\section{Synthesis of $\mathbf{N}$-chloroacetyl-piperidine-4-carboxamides. $\mathrm{N}$-chloroacetyl} piperidine-4-carbonyl chloride was prepared as a stock solution in dry DCM under an atmosphere of $\mathrm{N}_{2}$. In brief, deprotecting $\mathrm{N}$-Boc isonepecotic acid in $50 \%$ TFA in $\mathrm{DCM}(\mathrm{v} / \mathrm{v})$ at RT for $2 \mathrm{~h}$ yielded the corresponding TFA salt after evaporation of all volatiles. The crude TFA salt was then re-dissolved in DCM, treated with $\mathrm{Et}_{3} \mathrm{~N}$ (2 equiv.), followed by the addition of chloroacetic anhydride (1 equiv.). The reaction mixture was stirred overnight at RT, washed with water, the organic phase dried over $\mathrm{MgSO}_{4}$, filtered, and all volatiles removed by rotary evaporation. The crude $\mathrm{N}$ chloroacetyl piperidine-4-carboxylic acid was refluxed in excess neat $\mathrm{SOCl}_{2}$ (gas evolution and a colour change to red occurs) for $1 \mathrm{~h}$, followed by removal of excess $\mathrm{SOCl}_{2}$ in vacuum into a liquid nitrogen-cooled trap. The remaining residue was dried by rotary evaporation, placed under an atmosphere of nitrogen and dissolved in dry DCM to give a stock solution of $\sim 0.489 \mathrm{M}$ (based on theoretical yield over three steps), which was immediately used.

The corresponding amides were prepared by the addition of the acid chloride (1 equiv.) as a DCM solution to the pertinent amines (1 equiv.) in presence of pyridine (1 equiv.) in DCM. Heterogeneous reaction mixtures were treated with a minimal amount of dry DMF to achieve full solubility. After stirring the reaction mixtures overnight, the solvents were removed in by rotary evaporation, redissolved in $50 \%$ aq. MeCN (and a minimal amount of DMSO to achieve higher solubility), followed by purification by (semi-)preparative RP-HPLC in massdirected automatic mode or manually.

Synthesis of PepLites. HATU (1.5 equiv.), DIPEA (3.0 equiv.) and the acid starting material (1.5 equiv.) were dissolved in DMF $(3-6 \mathrm{~mL})$ and stirred together at room temperature for $10 \mathrm{~min}$. 3-Bromoprop-2-yn-1-amine hydrochloride was added and the reaction mixture was stirred at $40{ }^{\circ} \mathrm{C}$ overnight. The reaction mixture was allowed to cool to room temperature, diluted with EtOAc or DCM and washed with saturated aqueous sodium bicarbonate solution, brine and water. The organic layer was dried over $\mathrm{MgSO}_{4}$, filtered and evaporated to afford crude product. The crude product was then purified by either normal or reverse-phase chromatography.

tert-Butyl (3-bromoprop-2-yn-1-yl)carbamate. A solution of $\mathrm{KOH}$ (2.7 g, 48 $\mathrm{mmol})$ in water $(15 \mathrm{~mL})$ was added dropwise to a solution of $\mathrm{N}$-bocpropargylamine $(3.0 \mathrm{~g}, 19 \mathrm{mmol})$ in $\mathrm{MeOH}(45 \mathrm{~mL})$ at $0{ }^{\circ} \mathrm{C}$ under nitrogen. The resulting solution was stirred at $0{ }^{\circ} \mathrm{C}$ for $10 \mathrm{~min}$ then bromine $(1.1 \mathrm{~mL}, 21 \mathrm{mmol})$ was added dropwise. The reaction mixture was allowed to warm to room temperature and was stirred at room temperature for $24 \mathrm{~h}$. The reaction mixture was diluted with water and extracted with diethyl ether. The organic extracts were combined, dried over $\mathrm{MgSO}_{4}$ and evaporated to afford crude product. The crude product was purified by flash silica chromatography, elution gradient $0-10 \%$ EtOAc in petroleum ether. Pure fractions were evaporated to dryness to afford tert-Butyl (3-bromoprop-2-yn1 -yl)carbamate $(3.5 \mathrm{~g}, 79 \%)$ as a white solid. $R_{\mathrm{f}}=0.34(10 \%$ EtOAC in petroleum ether); m.p. $108-110^{\circ} \mathrm{C}$; IR $v_{\max }\left(\mathrm{cm}^{-1}\right) 3345,2982,2219,2121,2082 ;{ }^{1} \mathrm{H}$ NMR $(500 \mathrm{MHz}, \mathrm{DMSO}-d 6) \delta 1.39(\mathrm{~s}), 3.76(\mathrm{~d}, J=5.9 \mathrm{~Hz}), 7.30(\mathrm{~d}, J=6.1 \mathrm{~Hz}) ;{ }^{13} \mathrm{C}$ NMR $\left(126 \mathrm{MHz}\right.$, DMSO- $\left.d_{6}\right) \delta 28.63,30.89,43.44,78.46,78.81,155.69$; LCMS $m / z$ $\mathrm{ES}^{+}[\mathrm{M}-\mathrm{Boc}+\mathrm{H}]^{+}$133.9; HRMS calcd for $\mathrm{C}_{8} \mathrm{H}_{12}{ }^{79} \mathrm{BrNO}_{2} 255.9949[\mathrm{M}(\mathrm{Br})+\mathrm{Na}]^{+}$ found 256.0209. 
3-Bromoprop-2-yn-1-amine hydrochloride. tert-Butyl (3-bromoprop-2-yn-1-yl) carbamate $(1.1 \mathrm{~g}, 4.7 \mathrm{mmol})$ was dissolved in $4 \mathrm{M} \mathrm{HCl}$ in dioxane $(30 \mathrm{~mL})$. The reaction mixture was stirred at room temperature for $2 \mathrm{~h}$ then evaporated to dryness to afford 3-bromoprop-2-yn-1-amine hydrochloride $(0.79 \mathrm{~g}, 99 \%)$ as a yellow solid. m.p. $169^{\circ} \mathrm{C}$; IR $v_{\max }\left(\mathrm{cm}^{-1}\right) 2856,2629,2226,2121,2074 ;{ }^{1} \mathrm{H}$ NMR $\left(500 \mathrm{MHz}\right.$, DMSO- $\left.d_{6}\right) \delta 3.78(\mathrm{~s}, 2 \mathrm{H}), 8.48(\mathrm{~s}, 3 \mathrm{H}) ;{ }^{13} \mathrm{C}$ NMR $\left(126 \mathrm{MHz}\right.$, DMSO- $\left.d_{6}\right)$ $\delta 29.69,49.38,73.90 ; \mathrm{LCMS} \mathrm{m}^{2} \mathrm{z} \mathrm{ES}+[\mathrm{M}+\mathrm{H}]^{+}$134.0; HRMS calcd for $\mathrm{C}_{3} \mathrm{H}_{5}{ }^{79} \mathrm{BrN}$ $1339605[\mathrm{M}(\mathrm{Br})+\mathrm{H}]^{+}$found 133.9598.

\section{(2S,3R)-2-Acetamido-N-(3-bromoprop-2-yn-1-yl)-3-(tert-butoxy)butana-} mide. (2S, 3S)-2-Acetamido-N-(3-bromoprop-2-yn-1-yl)-3-(tert-butoxy)butanamide was synthesized according to General procedure A using $(2 S, 3 R)-2$ acetamido-3-(tert-butoxu)butanoic acid $(0.41 \mathrm{~g}, 1.9 \mathrm{mmol})$. The crude product was purified by flash silica chromatography, elution gradient $0-10 \% \mathrm{MeOH}$ in DCM. Pure fractions were evaporated to dryness to afford $(2 S, 3 S)$-2-acetamido- $N$ - (3bromoprop-2-yn-1-yl)-3-(tert-butoxy)butanamide $(0.20 \mathrm{~g}, 42 \%)$ as a white solid. $\mathrm{R}_{\mathrm{f}}=0.46\left(10 \% \mathrm{MeOH}\right.$ in DCM); mp: $180-183^{\circ} \mathrm{C}$; IR $v_{\max }\left(\mathrm{cm}^{-1}\right) 3271,3078$, $2969,2935,2222,2113 ;{ }^{1} \mathrm{H}$ NMR $\left(500 \mathrm{MHz}\right.$, Methanol- $\left.d_{4}\right) \delta 1.16(\mathrm{~d}, J=6.2,5.0$ $\mathrm{Hz}), 1.21(\mathrm{~s}, J=3.9 \mathrm{~Hz}, 9 \mathrm{H}), 2.01(\mathrm{~s}, 3 \mathrm{H}), 3.91-4.09(\mathrm{~m}, 3 \mathrm{H}), 4.32(\mathrm{~d}, J=7.5 \mathrm{~Hz}$, $1 \mathrm{H}) ;{ }^{13} \mathrm{C}$ NMR $\left(126 \mathrm{MHz}\right.$, Methanol- $\left.d_{4}\right) \delta 18.61,21.15,27.27,28.90,41.92,58.81$, $67.21,74.16,75.57,171.19,171.92 ;$ LCMS m/z ES $+[\mathrm{M}+\mathrm{H}]+333.2 ;$ HRMS calcd for $\mathrm{C}_{13} \mathrm{H}_{21}{ }^{79} \mathrm{BrN}_{2} \mathrm{O}_{3} 333.2260[\mathrm{M}(\mathrm{Br})+\mathrm{H}]^{+}$found 333.0808

\section{(2S,3R)-2-Acetamido-N-(3-bromoprop-2-yn-1-yl)-3-hydroxybutanamide} (threonine PepLite). (2S,3S)-2-Acetamido- $\mathrm{N}$-(3-bromoprop-2-yn-1-yl)-3-(tertbutoxy)butanamide ( $80 \mathrm{mg}, 0.24 \mathrm{mmol})$ was dissolved in anhydrous DCM $(20 \mathrm{~mL})$ and TFA $(10 \mathrm{~mL})$ and $0^{\circ} \mathrm{C}$ under nitrogen. The reaction mixture was allowed to warm to room temperature and was stirred at room temperature for $3 \mathrm{~h}$ then evaporated to dryness to afford crude product. The crude product was purified by flash silica chromatography, elution gradient $0-15 \% \mathrm{MeOH}$ in DCM. Pure fractions were evaporated to dryness to afford $(2 S, 3 S)$-2-acetamido- $N$-(3-bromoprop2-yn-1-yl)-3-hydroxybutanamide ( $38 \mathrm{mg}, 57 \%, 93 \% \mathrm{de}$ ) as a white solid. $\mathrm{R}_{\mathrm{f}}=0.34$ (10\% MeOH in DCM); mp: $189-192{ }^{\circ} \mathrm{C}$; IR $v_{\max }\left(\mathrm{cm}^{-1}\right) 3280,3085,2973,2924$, 2225, 2115; ${ }^{1} \mathrm{H}$ NMR $\left(500 \mathrm{MHz}\right.$, Methanol- $\left.d_{4}\right) \delta 1.21(\mathrm{~d}, J=6.4 \mathrm{~Hz}, 3 \mathrm{H}), 2.03(\mathrm{~s}$, $3 \mathrm{H}), 3.97-4.06(\mathrm{~m}, 3 \mathrm{H}), 4.33(\mathrm{~d}, J=6.5 \mathrm{~Hz}, 1 \mathrm{H}) ;{ }^{13} \mathrm{C} \mathrm{NMR}(126 \mathrm{MHz}$, Methanol-

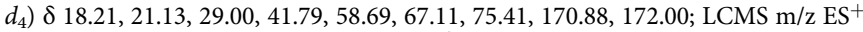
$[\mathrm{M}+\mathrm{H}]^{+}$277.1; HRMS calcd for $\mathrm{C}_{9} \mathrm{H}_{13}{ }^{79} \mathrm{BrN}_{2} \mathrm{O}_{3} 277.1180[\mathrm{M}(\mathrm{Br})+\mathrm{H}]^{+}$found 277.0182 .

\section{(S)-2-Acetamido- $N^{1}$-(3-bromoprop-2-yn-1-yl)succinimide (asparagine}

PepLite). (S)-2-Acetamido- $N^{1}$-(3-bromoprop-2-yn-1-yl)succinamide was synthesized according to General procedure A using $(s)$-2-acetamido-5-amino-5-oxobutanoic acid $(155 \mathrm{mg}, 0.89 \mathrm{mmol})$ and evaporating the reaction mixture to afford the crude product without aqueous work-up. The crude product was purified by flash silica chromatography, elution gradients $0-10 \% \mathrm{MeOH}$ in DCM. Pure fractions were evaporated to dryness to afford (S)-2-acetamido- $N^{1}$-(3-bromoprop-2-yn-1-yl) succinamide $(50 \mathrm{mg}, 30 \%)$ as a white solid. $\mathrm{R}_{\mathrm{f}}=0.18(10 \% \mathrm{MeOH}$ in DCM); $\mathrm{mp}$ : $173^{\circ} \mathrm{C}$ (decomp); IR $v_{\max }\left(\mathrm{cm}^{-1}\right) 3421,3277,3208,3072,2922,2226,2116 ; 1 \mathrm{H}$ NMR $(500 \mathrm{MHz}$, Methanol-d4) $\delta 1.99(\mathrm{~s}, 3 \mathrm{H}), 2.58-2.75(\mathrm{~m}, 2 \mathrm{H}), 3.98(\mathrm{~d}, J=1.4$ $\mathrm{Hz}, 2 \mathrm{H}), 4.71$ (dd, $J=7.6,5.7 \mathrm{~Hz}, 1 \mathrm{H}) ; 13 \mathrm{C}$ NMR $(126 \mathrm{MHz}$, Methanol-d4) $\delta$ $22.57,30.61,37.83,43.13,51.54,76.84,173.04,173.28,174.81 ; \mathrm{LCMS}^{\mathrm{m}} / \mathrm{z} \mathrm{ES}^{+}[\mathrm{M}$ $+\mathrm{H}]^{+}$290.2; HRMS calcd for $\mathrm{C}_{9} \mathrm{H}_{12}{ }^{79} \mathrm{BrN}_{3} \mathrm{O}_{3} 290.1170[\mathrm{M}(\mathrm{Br})+\mathrm{H}]^{+}$found 290.2265 .

Reporting summary. Further information on research design is available in the Nature Research Reporting Summary linked to this article.

\section{Data availability}

The coordinates and structure factors have been deposited in the Protein Data Bank. The accession codes are listed in Supplementary Data 2. Other data are available from the corresponding authors upon reasonable request.

Received: 5 June 2020; Accepted: 7 September 2020;

Published online: 07 October 2020

\section{References}

1. $\mathrm{Wu}, \mathrm{F}$. et al. A new coronavirus associated with human respiratory disease in China. Nature 579, 265 (2020)

2. Kucharski, A. J., Russell, T. W. \& Diamond, C. Early dynamics of transmission and control of COVID-19: a mathematical modelling study (vol 20, pg 553, 2020). Lancet Infect. Dis. 20, E79 (2020).

3. Zhu, N. et al. A novel coronavirus from patients with pneumonia in China, 2019. N. Engl. J. Med 382, 727-733 (2020).
4. Dong, E. S., Du, H. R. \& Gardner, L. An interactive web-based dashboard to track COVID-19 in real time. Lancet Infect. Dis. 20, 533-534 (2020).

5. Bermingham, A. et al. Severe respiratory illness caused by a novel coronavirus, in a patient transferred to the United Kingdom from the Middle East, September 2012. Eurosurveillance 17, 6-10 (2012).

6. Kuiken, T. et al. Newly discovered coronavirus as the primary cause of severe acute respiratory syndrome. Lancet 362, 263-270 (2003).

7. Zaki, A. M., van Boheemen, S., Bestebroer, T. M., Osterhaus, A. D. M. E. \& Fouchier, R. A. M. Isolation of a novel coronavirus from a man with pneumonia in Saudi Arabia. N. Engl. J. Med. 367, 1814-1820 (2012).

8. Bredenbeek, P. J. et al. The primary structure and expression of the 2 nd open reading frame of the polymerase gene of the coronavirus Mhv-A59 - a Highly conserved polymerase is expressed by an efficient ribosomal frameshifting mechanism. Nucleic Acids Res. 18, 1825-1832 (1990).

9. Thiel, V. et al. Mechanisms and enzymes involved in SARS coronavirus genome expression. J. Gen. Virol. 84, 2305-2315 (2003).

10. Hilgenfeld, R. From SARS to MERS: crystallographic studies on coronaviral proteases enable antiviral drug design. Febs J. 281, 4085-4096 (2014).

11. Ghosh, A. K. et al. Structure-based design,synthesis, and biological evaluation of peptidomimetic SARS-CoV 3CLpro inhibitors. Bioorg. Med. Chem. Lett. 17, 5876-5880 (2007).

12. Verschueren, K. H. G. et al. A structural view of the inactivation of the SARS coronavirus main proteinase by benzotriazole esters. Chem. Biol. 15, 597-606 (2008).

13. Yang, H. T. et al. Design of wide-spectrum inhibitors targeting coronavirus main proteases. PLoS Biol. 3, 1742-1752 (2005).

14. Yang, H. T. et al. The crystal structures of severe acute respiratory syndrome virus main protease and its complex with an inhibitor. Proc. Natl Acad. Sci. USA 100, 13190-13195 (2003).

15. Anand, K., Ziebuhr, J., Wadhwani, P., Mesters, J. R. \& Hilgenfeld, R. Coronavirus main proteinase (3CL(pro)) structure: basis for design of antiSARS drugs. Science 300, 1763-1767 (2003).

16. Hegyi, A. \& Ziebuhr, J. Conservation of substrate specificities among coronavirus main proteases. J. Gen. Virol. 83, 595-599 (2002).

17. Stadler, K. et al. SARS-Beginning to understand a new virus. Nat. Rev. Microbiol. 1, 209-218 (2003).

18. Xue, X. Y. et al. Structures of two coronavirus main proteases: implications for substrate binding and antiviral drug design. J. Virol. 82, 2515-2527 (2008).

19. Zhang, L. L. et al. Crystal structure of SARS-CoV-2 main protease provides a basis for design of improved alpha-ketoamide inhibitors. Science 368, 409-412 (2020).

20. Zhang, L. et al. alpha-Ketoamides as broad-spectrum inhibitors of coronavirus and enterovirus replication: structure-based design, synthesis, and activity assessment. J. Med. Chem. 63, 4562-4578 (2020).

21. Severson, W. E. et al. Development and validation of a high-throughput screen for inhibitors of SARS CoV and its application in screening of a 100,000compound library. J. Biomol. Screen 12, 33-40 (2007).

22. Thomas, S. E. et al. Structure-guided fragment-based drug discovery at the synchrotron: screening binding sites and correlations with hotspot mapping. Philos. T R Soc. A 377, 20180422 (2019).

23. Erlanson, D. A., Fesik, S. W., Hubbard, R. E., Jahnke, W. \& Jhoti, H. Twenty years on: the impact of fragments on drug discovery. Nat. Rev. Drug Discov. 15, 605-619 (2016).

24. Helliwell, J. R. \& Mitchell, E. P. Synchrotron radiation macromolecular crystallography: science and spin-offs. IUCrJ 2, 283-291 (2015).

25. Cox, O. B. et al. A poised fragment library enables rapid synthetic expansion yielding the first reported inhibitors of PHIP(2), an atypical bromodomain. Chem. Sci. 7, 2322-2330 (2016)

26. Collins, P. M. et al. Gentle, fast and effective crystal soaking by acoustic dispensing. Acta Crystallogr. Sect. D.-Struct. Biol. 73, 246-255 (2017).

27. Krojer, T. et al. The XChemExplorer graphical workflow tool for routine or large-scale protein-ligand structure determination. Acta Crystallogr. Sect. D.Struct. Biol. 73, 267-278 (2017).

28. Wright, N. D. et al. The low-cost, semi-automated shifter microscope stage transforms speed and robustness of manual protein crystal harvesting. Preprint at https://www.biorxiv.org/content/10.1101/2019.12.20.875674v1 (2019).

29. Resnick, E. et al. Rapid covalent-probe discovery by electrophile-fragment screening. J. Am. Chem. Soc. 141, 8951-8968 (2019).

30. Shi, J. H. \& Song, J. X. The catalysis of the SARS 3C-like protease is under extensive regulation by its extra domain. Febs J. 273, 1035-1045 (2006).

31. Jin, Z. et al. Structure of M(pro) from SARS-CoV-2 and discovery of its inhibitors. Nature 582, 289-293 (2020).

32. Nonoo, R. H., Armstrong, A. \& Mann, D. J. Kinetic template-guided tethering of fragments. ChemMedChem 7, 2082-2086 (2012).

33. Kathman, S. G., Xu, Z. \& Statsyuk, A. V. A fragment-based method to discover irreversible covalent inhibitors of cysteine proteases. J. Med. Chem. 57, 4969-4974 (2014) 
34. Kathman, S. G. et al. A small molecule that switches a ubiquitin ligase from a processive to a distributive enzymatic mechanism. J. Am. Chem. Soc. 137, 12442-12445 (2015).

35. Johansson, H. et al. Fragment-based covalent ligand screening enables rapid discovery of inhibitors for the RBR E3 ubiquitin ligase HOIP. J. Am. Chem. Soc. 141, 2703-2712 (2019).

36. Backus, K. M. et al. Proteome-wide covalent ligand discovery in native biological systems. Nature 534, 570-574 (2016).

37. Pearce, N. M. et al. A multi-crystal method for extracting obscured crystallographic states from conventionally uninterpretable electron density. Nat. Commun. 8, 15123 (2017).

38. Chou, C. Y. et al. Quaternary structure of the severe acute respiratory syndrome (SARS) coronavirus main protease. Biochem.-Us 43, 14958-14970 (2004).

39. Chen, S. et al. Residues on the dimer interface of SARS coronavirus 3C-like protease: dimer stability characterization and enzyme catalytic activity analysis. J. Biochem. 143, 525-536 (2008).

40. Hsu, W. C. et al. Critical assessment of important regions in the subunit association and catalytic action of the severe acute respiratory syndrome coronavirus main protease. J. Biol. Chem. 280, 22741-22748 (2005).

41. Barrila, J., Bacha, U. \& Freire, E. Long-range cooperative interactions modulate dimerization in SARS 3CL(pro). Biochemistry 45, 14908-14916 (2006).

42. Hu, T. C. et al. Two adjacent mutations on the dimer interface of SARS coronavirus 3C-like protease cause different conformational changes in crystal structure. Virology 388, 324-334 (2009).

43. Kuhn, B., Gilberg, E., Taylor, R., Cole, J. \& Korb, O. How significant are unusual protein-ligand interactions? Insights from database mining. J. Med. Chem. 62, 10441-10455 (2019).

44. Bauman, J. D., Harrison, J. J. E. K. \& Arnold, E. Rapid experimental SAD phasing and hot-spot identification with halogenated fragments. Iucrj 3, 51-60 (2016).

45. Wood, D. J. et al. FragLites-minimal, halogenated fragments displaying pharmacophore doublets. an efficient approach to druggability assessment and hit generation. J. Med. Chem. 62, 3741-3752 (2019).

46. Mons, E. et al. The alkyne moiety as a latent electrophile in irreversible covalent small molecule inhibitors of cathepsin K. J. Am. Chem. Soc. 141, 3507-3514 (2019).

47. Keeley, A., Abranyi-Balogh, P. \& Keseru, G. M. Design and characterization of a heterocyclic electrophilic fragment library for the discovery of cysteinetargeted covalent inhibitors. Medchemcomm 10, 263-267 (2019).

48. O’Reilly, M. et al. Crystallographic screening using ultra-low-molecularweight ligands to guide drug design. Drug Discov. Today 24, 1081-1086 (2019).

49. Keeley, A. et al. Heterocyclic electrophiles as new MurA inhibitors. Arch. Pharm.351, e1800184 (2018).

50. Fuller, N. et al. An improved model for fragment-based lead generation at AstraZeneca. Drug Discov. Today 21, 1272-1283 (2016).

51. Arrowsmith, C. H. et al. The promise and peril of chemical probes. Nat. Chem. Biol. 11, 536-541 (2015).

52. Ferla, M. P., Pagnamenta, A. T., Damerell, D., Taylor, J. C. \& Marsden, B. D. MichelaNglo: sculpting protein views on web pages without coding. Bioinformatics 36, 3268-3270 (2020).

53. Lee, W. H. et al. Interactive JIMD articles using the iSee concept: turning a new page on structural biology data. J. Inherit. Metab. Dis. 34, 565-567 (2011).

54. Pearce, N. M., Krojer, T. \& von Delft, F. Proper modelling of ligand binding requires an ensemble of bound and unbound states. Acta Crystallogr. D. Struct. Biol. 73, 256-266 (2017).

55. Singh, J., Petter, R. C., Baillie, T. A. \& Whitty, A. The resurgence of covalent drugs. Nat. Rev. Drug Discov. 10, 307-317 (2011).

56. Bauer, R. A. Covalent inhibitors in drug discovery: from accidental discoveries to avoided liabilities and designed therapies. Drug Discov. Today 20, 1061-1073 (2015).

57. De Cesco, S., Kurian, J., Dufresne, C., Mittermaier, A. K. \& Moitessier, N. Covalent inhibitors design and discovery. Eur. J. Med. Chem. 138, 96-114 (2017).

58. Zhang, T., Hatcher, J. M., Teng, M., Gray, N. S. \& Kostic, M. Recent advances in selective and irreversible covalent ligand development and validation. Cell Chem. Biol. 26, 1486-1500 (2019).

59. Baillie, T. A. Targeted covalent inhibitors for drug design. Angew. Chem. Int Ed. Engl. 55, 13408-13421 (2016).

60. Sirois, S., Hatzakis, G., Wei, D., Du, Q. \& Chou, K. C. Assessment of chemical libraries for their druggability. Comput. Biol. Chem. 29, 55-67 (2005).

61. Baell, J. B. \& Nissink, J. W. M. Seven year itch: pan-assay interference compounds (PAINS) in 2017-utility and limitations. ACS Chem. Biol. 13, 36-44 (2018).
62. Baell, J. B. \& Holloway, G. A. New substructure filters for removal of pan assay interference compounds (PAINS) from screening libraries and for their exclusion in bioassays. J. Med. Chem. 53, 2719-2740 (2010).

63. Winter, G. et al. DIALS: implementation and evaluation of a new integration package. Acta Crystallogr. Sect. D.-Struct. Biol. 74, 85-97 (2018).

64. Winter, G., Lobley, C. M. C. \& Prince, S. M. Decision making in xia2. Acta Crystallogr. D. 69, 1260-1273 (2013).

65. Evans, P. R. \& Murshudov, G. N. How good are my data and what is the resolution? Acta Crystallogr. D. 69, 1204-1214 (2013).

66. Emsley, P., Lohkamp, B., Scott, W. G. \& Cowtan, K. Features and development of Coot. Acta Crystallogr. D. 66, 486-501 (2010).

67. Murshudov, G. N. et al. REFMAC5 for the refinement of macromolecular crystal structures. Acta Crystallogr. Sect. D.-Struct. Biol. 67, 355-367 (2011).

68. Buster v. 2.10.13 (Cambridge, United Kingdom, 2017).

69. Downes, T. D. et al. Design and synthesis of 56 shape-diverse $3 \mathrm{D}$ fragments. Chemistry 26, 8969-8975 (2020).

70. Winter, G. et al. How best to use photons. Acta Crystallogr. D. Struct. Biol. 75, 242-261 (2019).

71. Winter, G. \& McAuley, K. E. Automated data collection for macromolecular crystallography. Methods 55, 81-93 (2011).

72. Kabsch, W. Integration, scaling, space-group assignment and post-refinement. Acta Crystallogr. D. 66, 133-144 (2010).

73. Vonrhein, C. et al. Data processing and analysis with the autoPROC toolbox. Acta Crystallogr. Sect. D.-Struct. Biol. 67, 293-302 (2011).

74. Keegan, R., Wojdyr, M., Winter, G. \& Ashton, A. DIMPLE: a difference map pipeline for the rapid screening of crystals on the beamline. Acta Crystallogr. A 71, S18-S18 (2015).

75. Long, F. et al. AceDRG: a stereochemical description generator for ligands. Acta Crystallogr. Sect. D.-Struct. Biol. 73, 112-122 (2017).

76. grade v. 1.2.19 (Global Phasing Ltd., Cambridge, United Kingdom, 2010).

\section{Acknowledgements}

We thank all the staff of Diamond Light Source for providing support and encouragement which allowed us to carry out this work during the COVID-19 lockdown. We also thank the Diamond MX group for their support and expertise, in particular David Aragão, Ralf Flaig, Dave Hall, Katherine McAuley and Mark Williams. We thank Clemens Vonrhein of GlobalPhasing for independent validation of the initially deposited models. We are grateful to AstraZeneca, Astex Pharmaceuticals, Lilly, Pfizer and Vernalis, University of York (T.D.D. and S.P.J.) and the EU (Horizon 2020 program, Marie Skłodowska-Curie grant agreement No. 675899, FRAGNET) (AK and HFK) for support The SGC is a registered charity (number 1097737) that receives funds from AbbVie, Bayer Pharma AG, Boehringer Ingelheim, Canada Foundation for Innovation, Eshelman Institute for Innovation, Genome Canada, Innovative Medicines Initiative (EU/EFPIA) [ULTRA-DD grant no. 115766], Janssen, Merck KGaA Darmstadt Germany, MSD, Novartis Pharma AG, Ontario Ministry of Economic Development and Innovation, Pfizer, São Paulo Research Foundation-FAPESP, Takeda, and Wellcome [106169/ZZ14/ Z]. N.L. is the incumbent of the Alan and Laraine Fischer Career Development Chair. N.L. would like to acknowledge funding from the Israel Science Foundation (grant no. 2462/19), The Israel Cancer Research Fund, the Israeli Ministry of Science Technology (grant no. 3-14763), the Moross Integrated Cancer Center and the Barry Sherman institute for Medicinal Chemistry. This research was supported by Nelson P. Sirotsky. N.L. is also supported by the Helen and Martin Kimmel Center for Molecular Design, Joel and Mady Dukler Fund for Cancer Research, the Estate of Emile Mimran and Virgin JustGiving, and the George Schwartzman Fund. FvD and GMK are grateful for the support of the Foreign Commonwealth and Development Office (UK). ABP received a Postdoctoral Fellowship from the Hungarian Science Foundation (PD124598).

\section{Author contributions}

M.A.W. initiated the project. N.L., F.V.D. and M.A.W. supervised and coordinated the research and analysed data. A.D., D.F., P.G., T.K., P.L., C.S-D., C.D.O., E.F., G.M. M.J.W., N.L., F.V.D. and M.A.W. writing original draft and review, editing of manuscript. A.D., D.F. and A.P. XChem sample preparation, crystallographic data collection, data analysis, P.G. and R.N.R. Chemical synthesis. T.K. Data analysis and PDB depositions. P.L. and C.S-D. Cloning, protein production, data analysis. C.D.O. crystallization, crystallographic data collection and data analysis. E.F. Electrophilic fragment screening and intact protein mass spectrometry. P.A-B. and A.K. synthesis of heterocyclic electrophiles. G.M.K. heterocyclic electrophile library design and review of manuscript. T.D.D, J.D.F, S.P.J., H.F.K. and P.O.B. designed, synthesised and curated the York 3-D fragments. M.J.W. Conception and design of PepLite hits and data analysis. G.D. synthesis of PepLites and data analysis. M.E.M.N and M.P.M Analysis of PepLite crystal structures, data analysis and review of manuscript. A.A, J.B-N., A.C, A.D, L.D., M.F., R.S., M.S. and C.W. Resources and validation. All authors read and approved the manuscript.

\section{Competing interests}

The authors declare no competing interests. 


\section{Additional information}

Supplementary information is available for this paper at https://doi.org/10.1038/s41467020-18709-w.

Correspondence and requests for materials should be addressed to N.L., F.v.D. or M.A.W.

Peer review information Nature Communications thanks Andrey Kovalevsky and the other, anonymous, reviewer(s) for their contribution to the peer review of this work. Peer reviewer reports are available.

Reprints and permission information is available at http://www.nature.com/reprints

Publisher's note Springer Nature remains neutral with regard to jurisdictional claims in published maps and institutional affiliations. (c) (i) Open Access This article is licensed under a Creative Commons Attribution 4.0 International License, which permits use, sharing, adaptation, distribution and reproduction in any medium or format, as long as you give appropriate credit to the original author(s) and the source, provide a link to the Creative Commons license, and indicate if changes were made. The images or other third party material in this article are included in the article's Creative Commons license, unless indicated otherwise in a credit line to the material. If material is not included in the article's Creative Commons license and your intended use is not permitted by statutory regulation or exceeds the permitted use, you will need to obtain permission directly from the copyright holder. To view a copy of this license, visit http://creativecommons.org/ licenses/by/4.0/.

(C) The Author(s) 2020 